\title{
Functional Dissection of Glutamatergic and GABAergic Neurons in the Bed Nucleus of the Stria Terminalis
}

\author{
Seong-Rae Kim and Sung-Yon Kim*
}

Institute of Molecular Biology and Genetics, Department of Chemistry, Seoul National University, Seoul 08826, Korea

*Correspondence: sungyonkim@snu.ac.kr

https://doi.org/10.14348/molcells.2021.0006

www.molcells.org

The bed nucleus of the stria terminalis (BNST)-a key part of the extended amygdala-has been implicated in the regulation of diverse behavioral states, ranging from anxiety and reward processing to feeding behavior. Among the host of distinct types of neurons within the BNST, recent investigations employing cell type- and projectionspecific circuit dissection techniques (such as optogenetics, chemogenetics, deep-brain calcium imaging, and the genetic and viral methods for targeting specific types of cells) have highlighted the key roles of glutamatergic and GABAergic neurons and their axonal projections. As anticipated from their primary roles in excitatory and inhibitory neurotransmission, these studies established that the glutamatergic and GABAergic subpopulations of the BNST oppositely regulate diverse behavioral states. At the same time, these studies have also revealed unexpected functional specificity and heterogeneity within each subpopulation. In this Minireview, we introduce the body of studies that investigated the function of glutamatergic and GABAergic BNST neurons and their circuits. We also discuss unresolved questions and future directions for a more complete understanding of the cellular diversity and functional heterogeneity within the BNST.

Keywords: bed nucleus of the stria terminalis, gammaaminobutyric acid, glutamate, neural circuitry

\section{INTRODUCTION}

The bed nucleus of the stria terminalis (BNST) is a basal forebrain structure situated posterior to the nucleus accumbens, anterior to the thalamus, medial to the dorsal striatum, and dorsal to the ventral pallidum and preoptic area (Alheid and Heimer, 1988). The BNST is a representative example of a highly heterogeneous and complex brain region, with 15-20 subregions harboring numerous types of neurons defined by cellular morphology (Ju et al., 1989), neurochemical makeup (Bota et al., 2012; Ju and Swanson, 1989; Kash et al., 2015; Poulin et al., 2009), electrophysiological properties (Daniel and Rainnie, 2016; Gungor and Paré, 2016), as well as their patterns of connectivity (Dong and Swanson, 2004; Dong et al., 2001; Lebow and Chen, 2016). The anatomical complexity of the BNST is well-matched with the many behavioral, autonomic, and endocrine functions it supports, which have been extensively discussed in the following excellent reviews: anxiety (Davis et al., 2010; Fox et al., 2015; Luyck et al., 2019; Robinson et al., 2019; Walker et al., 2009), stress (Daniel and Rainnie, 2016; Lebow and Chen, 2016; Radley and Johnson, 2018), aversive learning and memory (Goode and Maren, 2017; Gungor and Paré, 2016), addiction (Kash et al., 2015; Vranjkovic et al., 2017), social behavior (e.g., aggression, reproduction, and parenting) (Bayless and Shah, 2016; Flanigan and Kash, 2020), appetite control (Atasoy and Sternson, 2016; Zimmerman et al., 2017), as well as

Received 10 January, 2021; accepted 27 January, 2021; published online 17 February, 2021 
the control of heart rate and blood pressure (Crestani et al., 2013) and hormonal release (Radley and Johnson, 2018). Understanding how such diversity of functions is implemented by the heterogeneous constituents of the BNST remains to be a significant challenge.

A promising approach to tackle this problem-which turned out to be remarkably successful so far-is to investigate the anatomy and function of a specific neuron type defined by the expression of a marker gene (Huang and Zeng, 2013; Luo et al., 2018; Zeng and Sanes, 2017). This became possible with the development of neuron type-specific activity manipulation and imaging techniques, such as optogenetics and chemogenetics (Deisseroth, 2011; Roth, 2016), two-photon calcium imaging, and fiber photometry (Chen et al., 2013; Cui et al., 2013; Gunaydin et al., 2014; Svoboda and Yasuda, 2006), in conjunction with the advancement in genetic and viral methods that grant researchers reproducible access to a genetically defined subpopulation of neurons (Betley and Sternson, 2011; Huang and Zeng, 2013; Luo et al., 2018; Nectow and Nestler, 2020; Yizhar et al., 2011). Indeed, the genetic marker-based neuron type-specific investigations over the past decade yielded a striking expansion in our understanding of the structure and function of the BNST at the cellular level.

Among the exciting progress, in this Minireview, we systematically review the studies that employed this cutting-edge approach to specifically investigate glutamatergic and GABAergic neurons in the BNST and describe how each contributed to an updated view of the BNST function. We also discuss further remaining questions and ongoing attempts to overcome the limitations of this approach.

\section{SPECIFIC TARGETING OF GLUTAMATERGIC AND GABAERGIC BNST SUBPOPULATIONS}

Glutamate and gamma-aminobutyric acid (GABA) are the principal excitatory and inhibitory neurotransmitters in the brain, respectively. The anterior and dorsal parts of the BNST are mostly comprised of GABAergic neurons, while the pos- terior and ventral parts of the BNST contain significant numbers of both glutamatergic and GABAergic neurons (Poulin et al., 2009). To target glutamatergic or GABAergic BNST subpopulations, most studies used the Vglut2 or Vgat gene (that encodes vesicular glutamate transporter 2 or vesicular GABA transporter, respectively) as the genetic marker, respectively (Bhatti et al., 2020; Jennings et al., 2013a; 2013b), while some used Gad2 (encoding 65 kDa isoform of glutamic acid decarboxylase) to gain genetic access to GABAergic BNST neurons (Hao et al., 2019). Specifically, these studies used knock-in mouse lines expressing a bacterial recombinase Cre under the promoter of Vglut2, Vgat, or Gad2 in combination with viral tools that allow Cre-dependent expression of optogenetic or chemogenetic tools or fluorescent calcium indicators. Notably, all the studies have demonstrated that optogenetic stimulation of each neural population indeed evoked the anticipated excitatory or inhibitory responses in the postsynaptic neurons, confirming the tight correspondence between molecular and electrophysiological phenotypes.

\section{OPPOSITE REGULATION OF THE DIVERSE BEHAVIORAL STATES}

Consistent with the fact that glutamatergic and GABAergic neurons can excite or inhibit the postsynaptic neurons, studies have reported the opposite roles of these neurons in the BNST in distinct behaviors (Fig. 1). For example, optogenetic stimulation of the axon terminals of Vglut2-expressing neurons of the ventral BNST (vBNST) in the ventral tegmental area (VTA, a well-established center for reward processing and motivated behavior; Schultz et al., 1997) in behaving animals induced place avoidance (i.e., mice avoided the chamber associated with the optogenetic stimulation of this pathway) and anxiety-like behavior, whereas analogous terminal stimulation of vBNST ${ }^{\text {vgat }}$ neurons in the VTA supported place preference and reduced anxiety-like behavior (Jennings et al., 2013b). In congruence with their opposite causal functions, in vivo single-unit recordings from

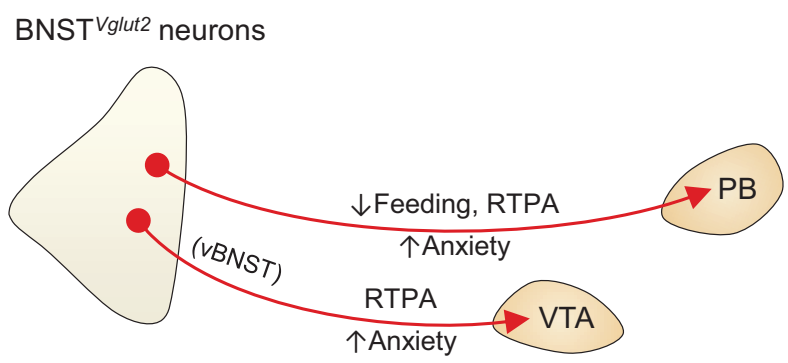

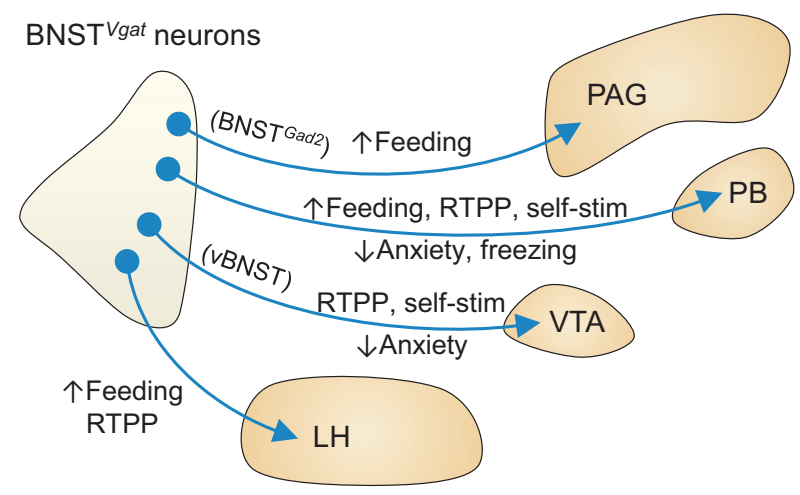

Fig. 1. Behavioral functions of the projections of GABAergic and glutamatergic BNST neurons. Summary of the causal roles of the efferent projections from BNST ${ }^{\text {Vgat }}$ and BNST ${ }^{G a d 2}$ neurons (left) and BNST ${ }^{\text {Vglut2 } 2}$ neurons (right). RTPP, real-time place preference; self-stim, self-stimulation; RTPA, real-time place aversion. Note that different circles and arrows do not indicate that separate subpopulations of neurons project to each target area. The anatomical relationship among the projections remains unclear, except that the BNST neurons projecting to the PAG and LH are distinct. 
VTA-projecting vBNST $T^{\text {Vglut2 }}$ or VBNST ${ }^{\text {vgat }}$ neurons revealed that these BNST subpopulations are mostly excited or inhibited by aversive stimuli, respectively. Optogenetic stimulation of either vBNST ${ }^{\text {Vglut2 }}$ or vBNST ${ }^{\text {Vgat }}$ neurons evoked corresponding excitatory or inhibitory postsynaptic responses in both tyrosine hydroxylase (TH)-expressing or non-expressing neurons (putative dopaminergic and non-dopaminergic neurons) in the VTA in an ex vivo electrophysiology setting, yet more responsive neurons were found among TH-negative neurons, suggesting that the control of non-dopaminergic VTA neurons may underlie the observed effects of the manipulation of vBNST ${ }^{\text {vglut2 }}$ and vBNST ${ }^{\text {vgat }}$ neurons.

Another study that showed the opposing roles of these populations focused on their projections to the parabrachial nucleus (PB), a small pontine structure that has been implicated in a number of functions including feeding (Kim et al., 2020; Palmiter, 2018). In this study, optogenetic stimulation of the axon terminals of BNST ${ }^{\text {Vglut2 }}$ neurons in the PB suppressed feeding in food-deprived mice, induced place avoidance, supported negative reinforcement of an operant behavior to turn off the stimulation, and increased anxiety-like behavior (Bhatti et al., 2020). In contrast, stimulating the terminals of BNST ${ }^{\text {vaat }}$ neurons increased food intake in ad libitum fed mice, induced place preference, supported positive reinforcement of an operant task to turn on the stimulation, had an anxiolytic effect, and suppressed freezing responses to a learned auditory threat signal. In line with their causal roles, fiber photometry recordings from the axon terminals of BNST $^{\text {Vglut2 }}$ neurons in the PB revealed that these terminals are activated upon footshock and inhibited during food intake, whereas the equivalent terminal recordings from BNST ${ }^{\text {vgat }}$ neurons showed that these are inhibited by footshock and activated during feeding behavior. Together, these studies suggest that glutamatergic and GABAergic BNST neurons might be oppositely regulated by motivationally salient stimuli, and can exert opposite influences on multiple behaviors (Bromberg-Martin et al., 2010; Janak and Tye, 2015).

\section{ANATOMICAL AND FUNCTIONAL HETEROGENEITY}

Notably, while the activation of both the BNST ${ }^{\text {Vgat }}$-PB and

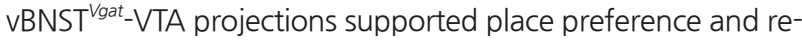
duced anxiety-like behaviors, only the activation of the former increased feeding behavior (Bhatti et al., 2020; Jennings et al., 2013a). Furthermore, a separate study showed that optogenetic stimulation of the projection from the anterior dorsal part of the BNST (adBNST, mostly composed of GABAergic neurons) to the VTA supports place preference, but does not affect anxiety-like behavior (Kim et al., 2013). Thus, the effects of activating BNST ${ }^{\text {vgat }}$ neurons on emotional valence, feeding, and anxiety can be dissociated by more refined targeting based on projection targets or subregional locations. As such, BNST ${ }^{\text {Vglut2 }}$ and BNST ${ }^{\text {Vgat }}$ neurons can be further subdivided into more functionally discrete subpopulations by other genetic markers, subregional distribution, and connectivity.

In addition to the BNST ${ }^{\text {Vat }}$-PB pathway, optogenetic stimulation of the projection from BNST ${ }^{\text {vgat }}$ neurons to the lateral hypothalamus (LH) (Jennings et al., 2013a) and the projection from BNST ${ }^{\text {Gad2 }}$ neurons to the periaqueductal gray (PAG)
(Hao et al., 2019) have also been shown to induce feeding behavior. In the LH projection study (Jennings et al., 2013a), the authors further demonstrated that optogenetic stimulation of the BNST ${ }^{\text {Vgat }}$-LH pathway induced place preference and intracranial self-stimulation (i.e., mice performed operant nosepoke responses to receive the brief optogenetic stimulation of this projection). Conversely, optogenetic inhibition of this projection induced place aversion, in addition to decreasing food consumption. Remarkably, self-stimulation of the BNST $^{\text {Vgat }}$-LH projection could be augmented or attenuated by food-deprivation or satiety, indicating that the hedonic value associated with the stimulation is dependent on the overall appetite level of the animals. Besides, optogenetic stimulation of the BNST ${ }^{\text {Vaat }}$-LH pathway induced a strong preference for high-fat food, suggesting that activation of this projection induces hedonic feeding as well as homeostatic feeding (Rossi and Stuber, 2018). The authors further demonstrated that the $\mathrm{LH}$ neurons that exhibit strong inhibitory postsynaptic currents (IPSCs) evoked upon optogenetic stimulation of the axon terminals of BNST ${ }^{\text {Vgat }}$ neurons abundantly expressed Vglut2, establishing that the main target of the BNST ${ }^{\text {Vaat }}-\mathrm{LH}$ pathway is $\mathrm{LH}^{\text {vglut2 }}$ neurons.

On the other hand, in the PAG projection study (Hao et al., 2019), the authors found that optogenetic stimulation of the axon terminals of BNST ${ }^{G a d 2}$ neurons in the ventrolateral PAG ( $V I P A G$ ) increased food intake in ad libitum fed mice, and inhibiting the same projection decreased feeding behavior in food-deprived mice. Importantly, the authors used retrograde tracers with two different colors to show that the vIPAG-projecting BNST neurons are distinct from the LH-projecting neurons. The overlap and separation among the many aforementioned projection-defined subpopulations of BNST neurons remain unclear. Determining the precise circuit organization has the potential to significantly advance our understanding of how glutamatergic and GABAergic BNST neurons regulate the feeding, and also reward-related and anxiety-like behaviors (Betley et al., 2013).

\section{CONCLUSION AND FUTURE PERSPECTIVES}

In this Minireview, we focused on the opposing roles of glutamatergic and GABAergic neurons in the BNST and covered the literature that used genetic markers to interrogate these subpopulations. Overall, GABAergic neurons in the BNST seem to induce a positive-valence, appetitive motivational drive, whereas glutamatergic neurons in the BNST elicit a state of negative valence, aversion, and anxiety. This offers a useful dichotomous perspective to understand the role of the BNST at a simpler level.

At the same time, these studies have also clearly demonstrated the functional heterogeneity within each population. This heterogeneity was revealed by single-unit recordings (Jennings et al., 2013b) and projection-specific activity recordings and manipulations (Bhatti et al., 2020; Hao et al., 2019; Jennings et al., 2013a). Recent studies targeting subpopulations of GABAergic BNST neurons also reinforce this notion. For example, neurons in the oval nucleus of the BNST defined by the expression of the prkcd gene, which is a subpopulation of GABAergic neurons, are activated upon various 
inflammatory signals (Wang et al., 2019). Notably, optogenetic stimulation of these neurons suppressed feeding, while the same manipulation did not have an effect on anxiety-like behavior, and failed to support conditioned place. These effects are in marked contrast to the orexigenic effects of the stimulation of the vBNST ${ }^{\text {Vgat }}$-VTA projection (Jennings et al.,

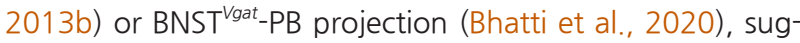
gestive of isolation of a more specific BNST subpopulation. Furthermore, another study targeting two subpopulations of GABAergic BNST neurons, characterized by the expression of the Crh and Cck genes, showed that each subpopulation is concentrated in the lateral and medial regions of the BNST, and activation of each drove aversion and approach to the stimulation-paired chamber, respectively (Giardino et al., 2018). Of note, chemogenetic activation of BNST ${ }^{\text {vgat }}$ neurons, which activates the $G_{q}$ protein-mediated signaling, was shown to increase anxiety-like behavior, suggesting that the recruitment of certain intracellular signaling pathways may also have specific effects in behavior (Mazzone et al., 2018).

The data from cell-type-specific interrogations provide invaluable building blocks for the construction of a detailed, realistic framework that can fully describe the inner workings of the multifunctional BNST circuitry. Undoubtedly, the field is moving forwards in this direction; such efforts are being accelerated by technical advances, including neuron type-specific activity manipulation and imaging techniques and genetic and viral methods as mentioned above, as well as the anatomical tissue processing techniques (Park et al., 2019; Seo et al., 2016; Ueda et al., 2020) and single-cell RNA-sequencing techniques (Armand et al., 2021; Tasic, 2018). In particular, the droplet-based single-cell RNA-sequencing approach has been recently applied to the BNST and revealed dozens of novel cell types (Moffitt et al., 2018; Rodriguez-Romaguera et al., 2020; Welch et al., 2019). Determining the precise functions of these subpopulations at the circuit and behavioral levels hold great promise for the comprehensive, mechanistic understanding of the diverse functions mediated by the BNST circuitry.

\section{ACKNOWLEDGMENTS}

We are grateful to Shanti Chang and Benjamin $\mathrm{H}$. Ahn for comments on the manuscript. This work was supported by National Research Foundation of Korea (NRF) grant funded by the Korea government (MSIT) (No. 2020R1C1C1003350) and Creative-Pioneering Researchers Program of Seoul National University.

\section{AUTHOR CONTRIBUTIONS}

S.R.K. and S.Y.K. wrote the manuscript.

\section{CONFLICT OF INTEREST}

The authors have no potential conflicts of interest to disclose.

\section{ORCID}

Seong-Rae Kim

Sung-Yon Kim

\section{REFERENCES}

Alheid, G.F. and Heimer, L. (1988). New perspectives in basal forebrain organization of special relevance for neuropsychiatric disorders: the striatopallidal, amygdaloid, and corticopetal components of substantia innominata. Neuroscience 27, 1-39.

Armand, E.J., Li, J., Xie, F., Luo, C., and Mukamel, E.A. (2021). Single-cell sequencing of brain cell transcriptomes and epigenomes. Neuron 109, 11-26.

Atasoy, D. and Sternson, S.M. (2016). Functional and anatomical dissection of feeding circuits. In Neuroendocrinology of Appetite, S.L. Dickson and J.G. Mercer, eds. (Hoboken: John Wiley \& Sons), pp. 112-133.

Bayless, D.W. and Shah, N.M. (2016). Genetic dissection of neural circuits underlying sexually dimorphic social behaviours. Philos. Trans. R. Soc. Lond. B Biol. Sci. 371, 20150109.

Betley, J.N., Cao, Z.F.H., Ritola, K.D., and Sternson, S.M. (2013). Parallel, redundant circuit organization for homeostatic control of feeding behavior. Cell 155, 1337-1350.

Betley, J.N. and Sternson, S.M. (2011). Adeno-associated viral vectors for mapping, monitoring, and manipulating neural circuits. Hum. Gene Ther. $22,669-677$.

Bhatti, D.L., Luskin, A.T., Pedersen, C.E., Mulvey, B., Oden-Brunson, H., Kimbell, K., Sawyer, A., Gereau, R.W., Dougherty, J.D., and Bruchas, M.R. (2020). Extended amygdala-parabrachial circuits alter threat assessment to regulate feeding. BioRxiv, https://doi.org/10.1101/2020.03.03.975193

Bota, M., Sporns, O., and Swanson, L.W. (2012). Neuroinformatics analysis of molecular expression patterns and neuron populations in gray matter regions: the rat BST as a rich exemplar. Brain Res. 1450, 174-193.

Bromberg-Martin, E.S., Matsumoto, M., and Hikosaka, O. (2010). Dopamine in motivational control: rewarding, aversive, and alerting. Neuron 68, 815-834

Chen, T.W., Wardill, T.J., Sun, Y., Pulver, S.R., Renninger, S.L., Baohan, A., Schreiter, E.R., Kerr, R.A., Orger, M.B., Jayaraman, V., et al. (2013). Ultrasensitive fluorescent proteins for imaging neuronal activity. Nature 499, 295-300.

Crestani, C.C., Alves, F.H., Gomes, F.V., Resstel, L.B., Correa, F.M., and Herman, J.P. (2013). Mechanisms in the bed nucleus of the stria terminalis involved in control of autonomic and neuroendocrine functions: a review. Curr. Neuropharmacol. 11, 141-159.

Cui, G., Jun, S.B., Jin, X., Pham, M.D., Vogel, S.S., Lovinger, D.M., and Costa, R.M. (2013). Concurrent activation of striatal direct and indirect pathways during action initiation. Nature 494, 238-242.

Daniel, S.E. and Rainnie, D.G. (2016). Stress modulation of opposing circuits in the bed nucleus of the stria terminalis. Neuropsychopharmacology 41, 103-125.

Davis, M., Walker, D.L., Miles, L., and Grillon, C. (2010). Phasic vs sustained fear in rats and humans: role of the extended amygdala in fear vs anxiety. Neuropsychopharmacology 35, 105-135.

Deisseroth, K. (2011). Optogenetics. Nat. Methods 8, 26-29.

Dong, H.W., Petrovich, G.D., and Swanson, L.W. (2001). Topography of projections from amygdala to bed nuclei of the stria terminalis. Brain Res. Brain Res. Rev. 38, 192-246.

Dong, H.W. and Swanson, L.W. (2004). Organization of axonal projections from the anterolateral area of the bed nuclei of the stria terminalis. J. Comp. Neurol. 468, 277-298.

Flanigan, M.E. and Kash, T.L. (2020). Coordination of social behaviors by the bed nucleus of the stria terminalis. Eur. J. Neurosci. 2020 Oct 2 [Epub]. https://doi.org/10.1111/ejn.14991

Fox, A.S., Oler, J.A., Tromp, D.P.M., Fudge, J.L., and Kalin, N.H. (2015). Extending the amygdala in theories of threat processing. Trends Neurosci. 38, 319-329. 
Giardino, W.J., Eban-Rothschild, A., Christoffel, D.J., Li, S.B., Malenka, R.C., and de Lecea, L. (2018). Parallel circuits from the bed nuclei of stria terminalis to the lateral hypothalamus drive opposing emotional states. Nat. Neurosci. 21, 1084-1095.

Goode, T.D. and Maren, S. (2017). Role of the bed nucleus of the stria terminalis in aversive learning and memory. Learn. Mem. 24, 480-491.

Gunaydin, L.A., Grosenick, L., Finkelstein, J.C., Kauvar, I.V., Fenno, L.E., Adhikari, A., Lammel, S., Mirzabekov, J.J, Airan, R.D., Zalocusky, K.A., et al. (2014). Natural neural projection dynamics underlying social behavior. Cell $157,1535-1551$

Gungor, N.Z. and Paré, D. (2016). Functional heterogeneity in the bed nucleus of the stria terminalis. J. Neurosci. 36, 8038-8049.

Hao, S., Yang, H., Wang, X., He, Y., Xu, H., Wu, X., Pan, L., Liu, Y., Lou, H., Xu, $H_{.}$, et al. (2019). The lateral hypothalamic and BNST GABAergic projections to the anterior ventrolateral periaqueductal gray regulate feeding. Cell Rep. 28, 616-624.e5.

Huang, J.Z. and Zeng, H. (2013). Genetic approaches to neural circuits in the mouse. Annu. Rev. Neurosci. 36, 183-215.

Janak, P.H. and Tye, K.M. (2015). From circuits to behaviour in the amygdala. Nature 517, 284-292.

Jennings, J.H., Rizzi, G., Stamatakis, A.M., Ung, R.L., and Stuber, G.D. (2013a). The inhibitory circuit architecture of the lateral hypothalamus orchestrates feeding. Science 341, 1517-1521.

Jennings, J.H., Sparta, D.R., Stamatakis, A.M., Ung, R.L., Pleil, K.E., Kash, T.L., and Stuber, G.D. (2013b). Distinct extended amygdala circuits for divergent motivational states. Nature 496, 224-228.

Ju, G. and Swanson, L.W. (1989). Studies on the cellular architecture of the bed nuclei of the stria terminalis in the rat: I. cytoarchitecture. J. Comp. Neurol. 280, 587-602.

Ju, G., Swanson, L.W., and Simerly, R.B. (1989). Studies on the cellular architecture of the bed nuclei of the stria terminalis in the rat: II. chemoarchitecture. J. Comp. Neurol. 280, 603-621.

Kash, T.L., Pleil, K.E., Marcinkiewcz, C.A., Lowery-Gionta, E.G., Crowley, N., Mazzone, C., Sugam, J., Hardaway, J.A., and McElligott, Z.A. (2015). Neuropeptide regulation of signaling and behavior in the BNST. Mol. Cells 38, 1-13.

Kim, D.Y., Heo, G., Kim, M., Kim, H., Jin, J.A., Kim, H.K., Jung, S., An, M., Ahn, B.H., Park, J.H., et al. (2020). A neural circuit mechanism for mechanosensory feedback control of ingestion. Nature 580, 376-380.

Kim, S.Y., Adhikari, A., Lee, S.Y., Marshel, J.H., Kim, C.K., Mallory, C.S., Lo, M., Pak, S., Mattis, J., Lim, B.K., et al. (2013). Diverging neural pathways assemble a behavioural state from separable features in anxiety. Nature 496, 219-223.

Lebow, M.A. and Chen, A. (2016). Overshadowed by the amygdala: the bed nucleus of the stria terminalis emerges as key to psychiatric disorders. Mol. Psychiatry 21, 450-463.

Luo, L., Callaway, E.M., and Svoboda, K. (2018). Genetic dissection of neural circuits: a decade of progress. Neuron 98, 256-281.

Luyck, K., Goode, T.D., Lee Masson, H., and Luyten, L. (2019). Distinct activity patterns of the human bed nucleus of the stria terminalis and amygdala during fear learning. Neuropsychol. Rev. 29, 181-185.

Mazzone, C.M., Pati, D., Michaelides, M., DiBerto, J., Fox, J.H., Tipton, G., Anderson, C., Duffy, K., McKlveen, J.M., Hardaway, J.A., et al. (2018). Acute engagement of $\mathrm{G} q$-mediated signaling in the bed nucleus of the stria terminalis induces anxiety-like behavior. Mol. Psychiatry 23, 143-153.

Moffitt, J.R., Bambah-Mukku, D., Eichhorn, S.W., Vaughn, E., Shekhar, K., Perez, J.D., Rubinstein, N.D., Hao, J., Regev, A., Dulac, C., et al. (2018). Molecular, spatial, and functional single-cell profiling of the hypothalamic preoptic region. Science 362, eaau5324.

Nectow, A.R. and Nestler, E.J. (2020). Viral tools for neuroscience. Nat. Rev. Neurosci. 21, 669-681.

Palmiter, R.D. (2018). The parabrachial nucleus: CGRP neurons function as a general alarm. Trends Neurosci. 41, 280-293.

Park, H.E., Choi, D., Park, J.S., Sim, C., Park, S., Kang, S., Yim, H., Lee, M., Kim, J., Pac, J., et al. (2019). Scalable and isotropic expansion of tissues with simply tunable expansion ratio. Adv. Sci. (Weinh.) 6, 1901673.

Poulin, J.F., Arbour, D., Laforest, S., and Drolet, G. (2009). Neuroanatomical characterization of endogenous opioids in the bed nucleus of the stria terminalis. Prog. Neuropsychopharmacol. Biol. Psychiatry 33, 1356-1365.

Radley, J.J. and Johnson, S.B. (2018). Anteroventral bed nuclei of the stria terminalis neurocircuitry: towards an integration of HPA axis modulation with coping behaviors - Curt Richter Award Paper 2017. Psychoneuroendocrinology 89, 239-249.

Robinson, O.J., Pike, A.C., Cornwell, B., and Grillon, C. (2019). The translational neural circuitry of anxiety. J. Neurol. Neurosurg. Psychiatry 90 , 1353-1360.

Rodriguez-Romaguera, J., Ung, R.L., Nomura, H., Otis, J.M., Basiri, M.L., Namboodiri, V.M.K., Zhu, X., Robinson, J.E., van den Munkhof, H.E., McHenry, J.A., et al. (2020). Prepronociceptin-expressing neurons in the extended amygdala encode and promote rapid arousal responses to motivationally salient stimuli. Cell Rep. 33, 108362.

Rossi, M.A. and Stuber, G.D. (2018). Overlapping brain circuits for homeostatic and hedonic feeding. Cell Metab. 27, 42-56.

Roth, B.L. (2016). DREADDs for neuroscientists. Neuron 89, 683-694.

Schultz, W., Dayan, P., and Montague, P.R. (1997). A neural substrate of prediction and reward. Science 275, 1593-1599.

Seo, J., Choe, M., and Kim, S.Y. (2016). Clearing and labeling techniques for large-scale biological tissues. Mol. Cells 39, 439-446.

Svoboda, K. and Yasuda, R. (2006). Principles of two-photon excitation microscopy and its applications to neuroscience. Neuron 50, 823-839.

Tasic, B. (2018). Single cell transcriptomics in neuroscience: cell classification and beyond. Curr. Opin. Neurobiol. 50, 242-249.

Ueda, H.R., Ertürk, A., Chung, K., Gradinaru, V., Chédotal, A., Tomancak, P., and Keller, P.J. (2020). Tissue clearing and its applications in neuroscience. Nat. Rev. Neurosci. 21, 61-79.

Vranjkovic, O., Pina, M., Kash, T.L., and Winder, D.G. (2017). The bed nucleus of the stria terminalis in drug-associated behavior and affect: a circuit-based perspective. Neuropharmacology 122, 100-106.

Walker, D.L., Miles, L.A., and Davis, M. (2009). Selective participation of the bed nucleus of the stria terminalis and CRF in sustained anxiety-like versus phasic fear-like responses. Prog. Neuropsychopharmacol. Biol. Psychiatry 33, 1291-1308

Wang, Y., Kim, J., Schmit, M.B., Cho, T.S., Fang, C., and Cai, H. (2019). A bed nucleus of stria terminalis microcircuit regulating inflammation-associated modulation of feeding. Nat. Commun. 10, 2769.

Welch, J.D., Kozareva, V., Ferreira, A., Vanderburg, C., Martin, C., and Macosko, E.Z. (2019). Single-cell multi-omic integration compares and contrasts features of brain cell identity. Cell 177, 1873-1887.e17.

Yizhar, O., Fenno, L.E., Davidson, T.J., Mogri, M., and Deisseroth, K. (2011). Optogenetics in neural systems. Neuron 71, 9-34.

Zeng, H. and Sanes, J.R. (2017). Neuronal cell-type classification: challenges, opportunities and the path forward. Nat. Rev. Neurosci. 18, 530-546.

Zimmerman, C.A., Leib, D.E., and Knight, Z.A. (2017). Neural circuits underlying thirst and fluid homeostasis. Nat. Rev. Neurosci. 18, 459-469. 